\title{
Mental health after paediatric concussion: a systematic review and meta-analysis
}

\author{
Alice Gornall (D) , 1,2 Michael Takagi (D) , 1,2,3 Thilanka Morawakage, ${ }^{2}$ Xiaomin Liu, $^{2}$ \\ Vicki Anderson (1) 2,3,4
}

- Additional supplemental material is published online only. To view, please visit the journal online (http://dx.doi. org/10.1136/bjsports-2020103548).

${ }^{1}$ Psychological Sciences, Monash University Faculty of Medicine Nursing and Health Sciences, Clayton, Victoria, Australia ${ }^{2}$ Brain and Mind Research, Clinical Sciences Theme, Murdoch Children's Research Institute, Parkville, Victoria, Australia

${ }^{3}$ Psychology, The University of Melbourne, Melbourne School of Psychological Sciences, Melbourne, Victoria, Australia ${ }^{4}$ Psychology Service, The Royal Children's Hospital, Mebourne, Victoria, Australia

Correspondence to Professor Vicki Anderson, Brain and Mind Research, Murdoch Children's Research Institute Clinical Sciences Theme, Parkville, VIC 3052, Australia; vicki.anderson@rch.org.au

Accepted 20 March 2021 Published Online First 29 April 2021

\begin{abstract}
Objective This systematic review and meta-analysis sought to rigorously examine mental health outcomes following paediatric concussion. To date, heterogeneous findings and methodologies have limited clinicians' and researchers' ability to meaningfully synthesise existing literature. In this context, there is a need to clarify mental health outcomes in a homogeneous sample, controlling for key methodological differences and applying a consistent definition of concussion across studies.
\end{abstract}

Design Systematic review and meta-analysis.

Data sources We searched Medline, Embase, PsycINFO, CINAHL, SportDiscus, Scopus and PubMed.

Eligibility Peer-reviewed studies published between 1980 and June 2020 that prospectively examined mental health outcomes after paediatric concussion, defined as per the Berlin Consensus Statement on Concussion in Sport.

Results Sixty-nine articles characterising 60 unique samples met inclusion criteria, representing 89114 children with concussion. Forty articles (33 studies) contributed to a random effects meta-analysis of internalising (withdrawal, anxiety, depression, post-traumatic stress), externalising (conduct problems, aggression, attention, hyperactivity) and total mental health difficulties across three time points postinjury (acute, persisting and chronic). Overall, children with concussion ( $n=6819$ ) experienced significantly higher levels of internalising ( $g=0.41-0.46$ ), externalising ( $g=0.25-0.46$ ) and overall mental health difficulties compared with controls ( $g=0.18-0.49 ; n=56271)$, with effects decreasing over time. Summary/conclusions Our review highlights that mental health is central to concussion recovery. Assessment, prevention and intervention of mental health status should be integrated into standard follow-up procedures. Further research is needed to clarify the mechanisms underlying observed relationships between mental health, postconcussion symptoms and other psychosocial factors. Results suggest that concussion may both precipitate and exacerbate mental health difficulties, thus impacting delayed recovery and psychosocial outcomes.

\section{INTRODUCTION}

Concussion is a growing public health concern with onethird of children experiencing a head injury before age 13 years. ${ }^{1}$ Despite the incidence of concussion among children and adolescents, identifying young people at risk of ongoing difficulties after concussion remains a prominent challenge in the field. ${ }^{2}$ Further, evidence shows that children take twice as long to recover from concussion than adults, with one in four children experiencing symptoms beyond 1 month post-injury, defined here as delayed recovery. ${ }^{3-5}$ Important differences in the mechanism and context blur the differentiation of sports and non-sports concussion in children and limit the translation of adult findings to paediatric concussion. ${ }^{6}$ For example, paediatric concussions are less likely to be sports related and more likely to be due to falls sustained in play or leisure, and more likely to occur in the home or in non-competitive activities. Child and adolescent athletes are also distinguished from older athletes along several dimensions, including biomechanical and pathophysiological responses, and contextual demands (eg, learning vs work). ${ }^{8}$

After concussion, due to either sport or non-sport injuries, children and adolescents are susceptible to a broad range of physical, behavioural, emotional, cognitive, somatic and sleep-related symptoms, ${ }^{9}$ many of which overlap with common mental health symptoms. Indeed, evidence suggests that post-concussive symptoms (PCS) correlate highly with internalising symptoms such as anxiety ${ }^{10}$ and depression, ${ }^{11}{ }^{12}$ with premorbid mental illness identified as a key risk factor for delayed recovery. ${ }^{13}$ Mental health screening may provide an avenue for early detection and targeted interventions for children at risk of delayed recovery due to mental health symptoms. Despite the potential role that these behavioural and emotional disturbances may play in delayed recovery, few studies have rigorously examined this relationship. ${ }^{14}$

Research designs employed in studies investigating mental health outcomes after paediatric concussion have, to date, been heterogeneous, limiting opportunities for qualitative and quantitative synthesis. ${ }^{14}$ Variable definitions of concussion adopted across studies are a key contributing factor to this heterogeneity. Further, combining traumatic brain injury (TBI) severity groups for analysis, unclear eligibility criteria (eg, pre-injury mental health status), differences in recruitment methods (eg, inpatient, outpatient, community), nature of comparison groups and timing of outcome measurement all contribute to variability. Not surprisingly, results are also inconsistent with some studies reporting persistent mental health symptoms, ${ }^{15-18}$ while others report no such findings. ${ }^{19} 20$

Despite methodological challenges, several robust findings are reported in the literature. Female adolescents are particularly vulnerable to internalising difficulties such as depression, anxiety and withdrawal after concussion. $^{21}$ Meanwhile, externalising behaviours, such as conduct problems, attention difficulties, hyperactivity and temper problems are more common among younger children. ${ }^{22}$ Whether these findings reflect risk factors associated with participant factors, such as age and sex, versus design characteristics require further exploration.

Two previous reviews have examined psychiatric outcomes from paediatric mild TBI (mTBI), however, both merged concussion, mTBI and complicated mTBI 


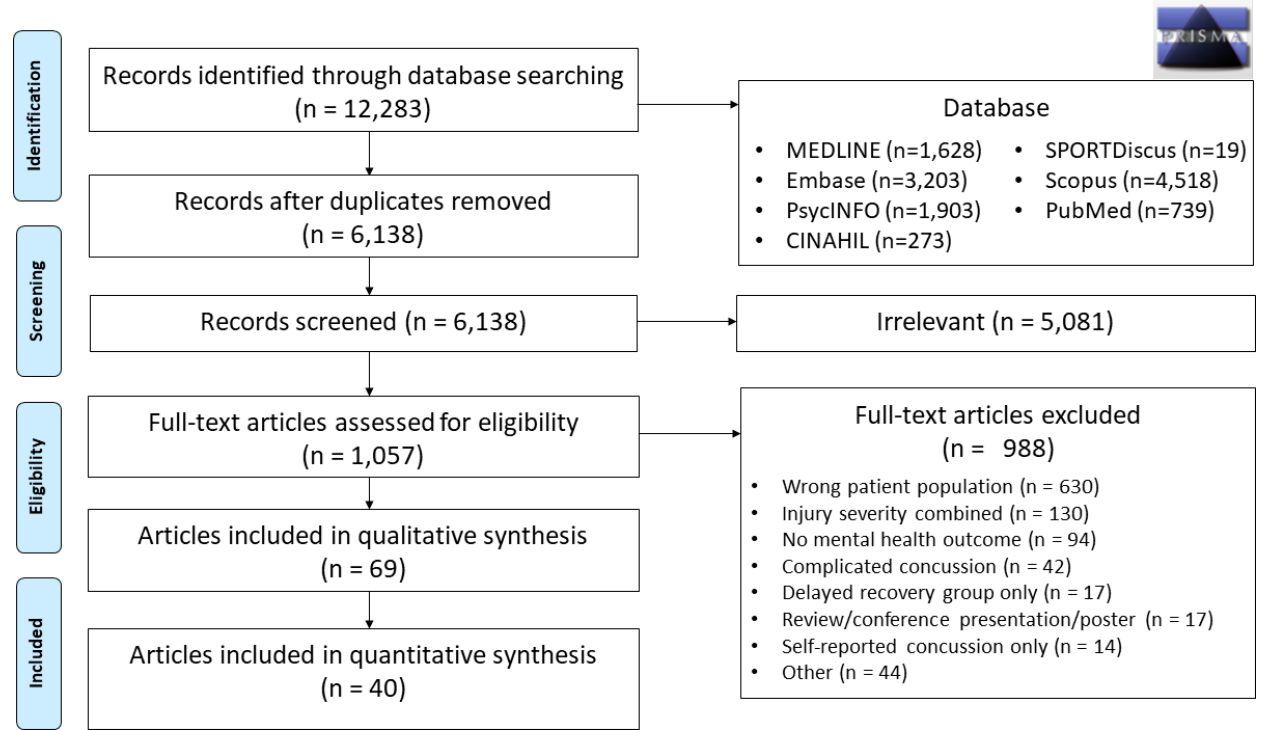

Figure 1 Preferred Reporting Items for Systematic Reviews and Meta-Analyses flow chart.

in their syntheses. ${ }^{14} 23$ These reviews emphasise high levels of variability in study findings, concluding that evidence for mental health difficulties following paediatric mTBI is inconclusive. Nonetheless, several important distinctions between these diagnoses should be acknowledged. In mTBI, recovery profiles for injuries with and without structural brain abnormalities display distinct characteristics, with structural injuries associated with slower recovery and poorer functional outcomes across cognitive, academic and social domains than when no structural injury is detected. ${ }^{24-31}$ In the present study, we have applied the definition of concussion from the Berlin Consensus Statement of Concussion in Sport (CISG), specifically 'an acute functional disturbance coupled with post-concussion symptoms, but without evidence of structural brain injury and generally associated with the expectation of better recovery than mTBI'. While this definition was derived with a focus on sports concussion, it refers to injury consequences and is equally applicable to concussions sustained outside organised sport.

The objective of this review was to systematically examine prospective mental health outcomes following paediatric concussion, applying a consistent definition of concussion across studies. Specifically, we sought to address the following research questions: (1) How common are mental health difficulties after paediatric concussion? (2) What factors predict mental health following paediatric concussion? (3) How do mental health problems interact with recovery from concussion? and (4) How does mental health status change with time post-injury? We conducted a meta-analysis to determine if children experienced elevated mental health difficulties after concussion compared with controls.

\section{MATERIALS AND METHODS}

\section{Protocol registration}

The review protocol was registered with the International Prospective Register of Systematic Reviews (\#147741).

\section{Methods}

This systematic review was conducted according to Preferred Reporting Items for Systematic Reviews and Meta-Analyses guidelines and identified articles published from 1980 to June 2020 using the search strategy shown in online supplemental table 1 . This time frame was selected due to the considerable changes in the understanding of concussion over the past 40 years and the large number of articles identified in preliminary searches. The final search was conducted in June 2020. Studies identified through systematic searches of Medline, Embase, PsycINFO, CINAHL and SportDiscus. Keyword only searches were conducted through Scopus and PubMed to identify articles not captured via the above sources. Two postgraduate authors (AG, TM) independently screened titles and abstracts using Covidence Systematic Review Software. ${ }^{32}$ Articles identified as relevant in this phase were reviewed in full text (AG, XL), according to the eligibility criteria outlined below. Reviewers were blind to one another's decision at the time of voting. Any discrepancies highlighted by the software were resolved by consensus, and a third senior clinical researcher (MT) was consulted if additional expertise was required.

\section{Eligibility criteria}

This review included peer-reviewed articles reporting prospective mental health outcomes following concussion in paediatric populations (age 0-18 years at time of injury). To increase the application of present findings to inform policy and practice, this review considered quantitative, qualitative and mixed-methods studies. Quantitative studies included observational designs, prospective and retrospective cohort studies, case-control studies and analytical cross-sectional studies. Qualitative studies included, but were not limited to, phenomenology, grounded theory, ethnography and action research. Mixed-method studies were considered if data from the quantitative or qualitative components could be extracted.

Concussion was defined according to the Berlin CISG Criteria and required diagnosis by a health professional or administration of a clinically validated measure. ${ }^{9}$ The Berlin CISG Criteria were developed using rigorous methodology, including systematic review of the literature and expert panel consensus. Studies were eligible for inclusion if the concussion definition was consistent with the Berlin CISG Criteria, regardless of whether or not these criteria were explicitly cited.

Seventeen authors were contacted to clarify whether participants satisfied present criteria for concussion. Intervention studies that did not report prospective mental health outcomes for a non-intervention concussion group, studies that combined injury severities (eg, did not differentiate between concussion 


\section{Acute Internalising Problems ( $<3$ months)}

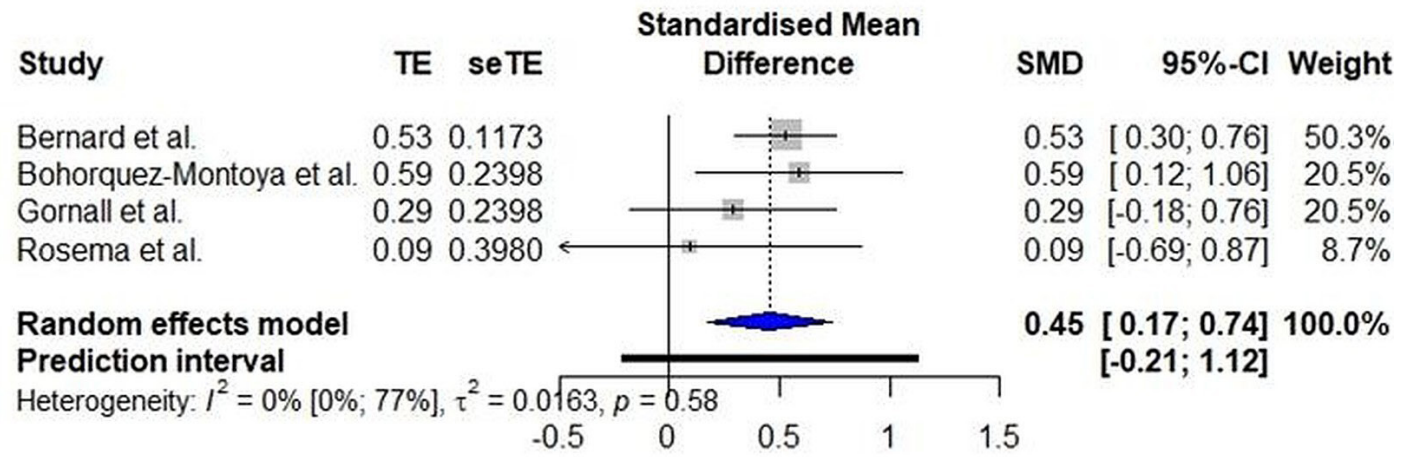

\section{Persisting Internalising Problems (3-12 months)}

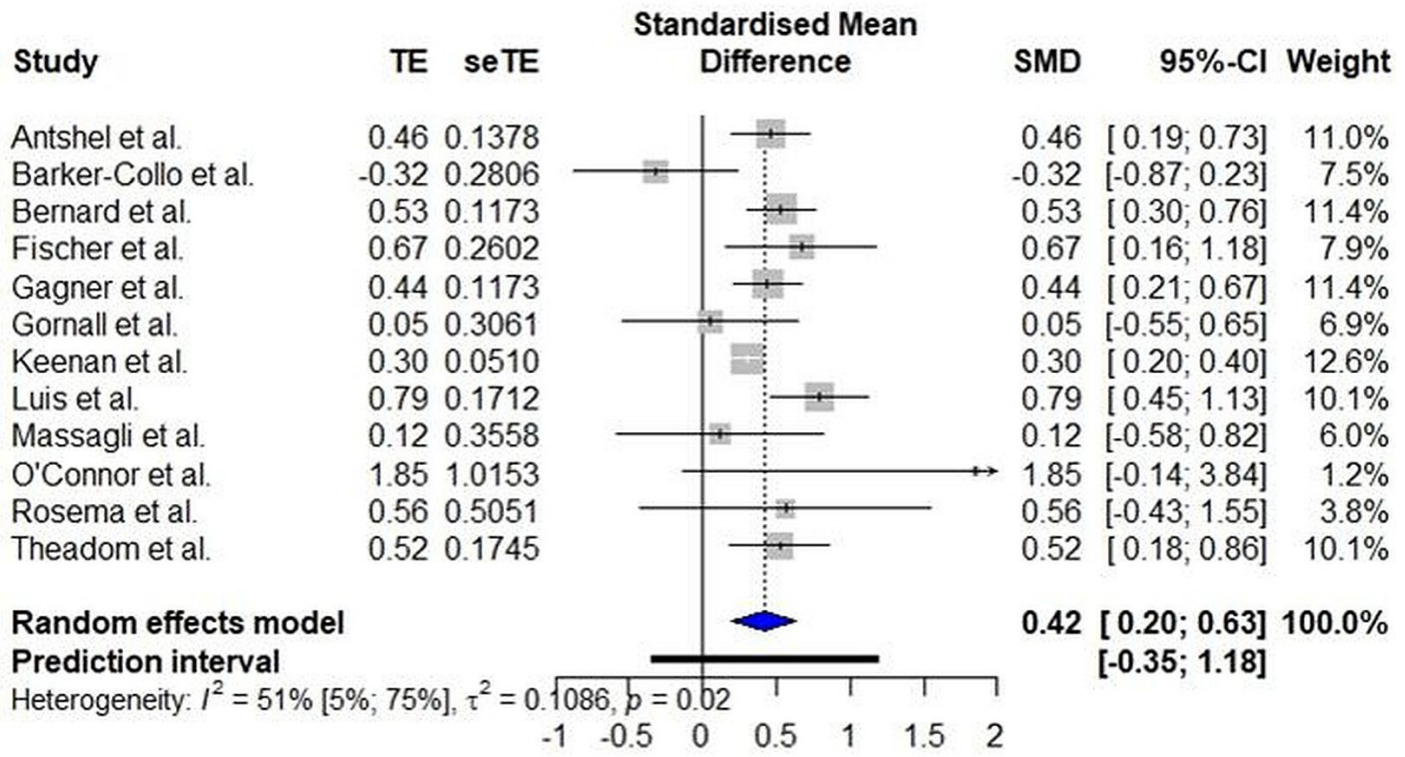

\section{Chronic Internalising Problems (>12 months)}

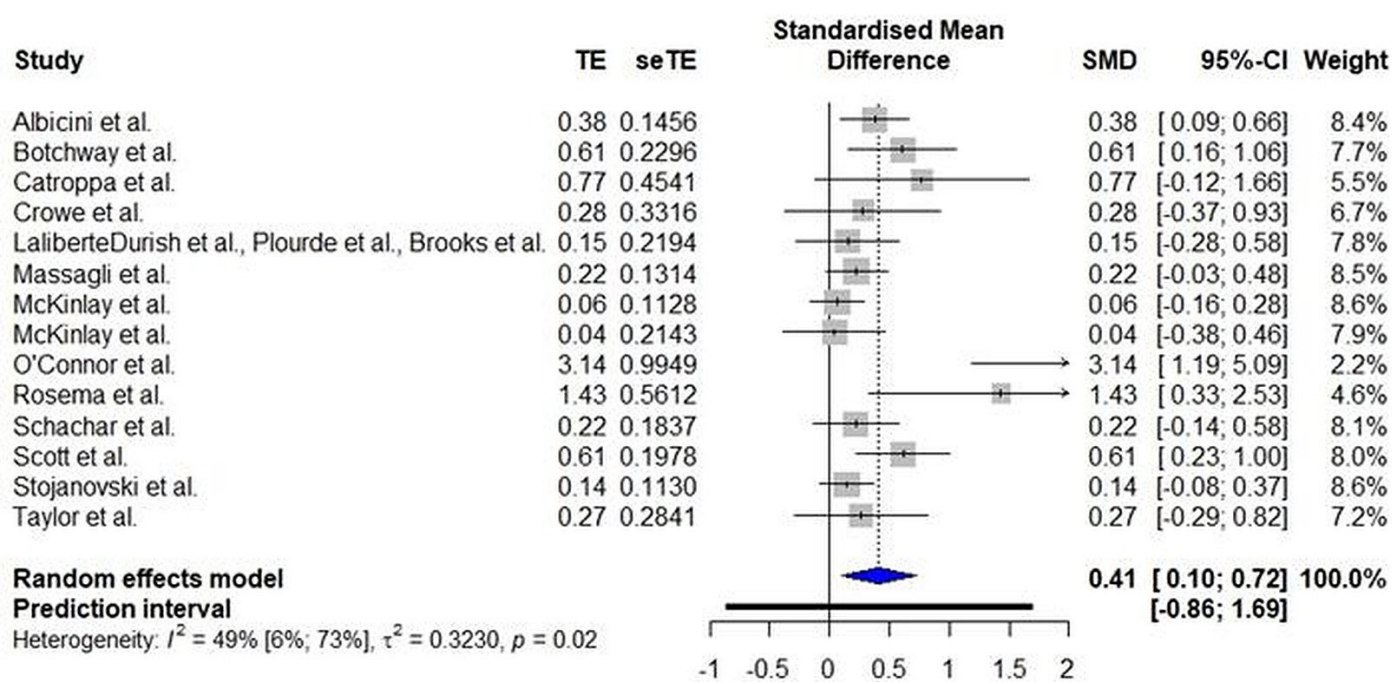

Figure 2 Standard mean difference (SMD), treatment effect (TE) and standard error (seTE) of acute, persisting and chronic internalising problems after paediatric concussion. 


\section{Acute Externalising Problems ( $<3$ months)}

\begin{tabular}{|c|c|c|}
\hline Study & TE seTE & $\begin{array}{l}\text { Standardised Mean } \\
\text { Difference }\end{array}$ \\
\hline Bernard et al. & 0.310 .1684 & $\because$ \\
\hline Gornall et al. & 0.530 .3112 & \\
\hline Rosema et al. & 0.440 .4082 & \\
\hline \multicolumn{3}{|c|}{ Random effects model } \\
\hline
\end{tabular}

SMD $\quad 95 \%-\mathrm{Cl}$ Weight

$0.31[-0.02 ; 0.64] \quad 67.5 \%$

$0.53[-0.08 ; 1.14] \quad 20.5 \%$

$0.44[-0.36,1.24] \quad 12.0 \%$

$0.37[0.09 ; 0.65] 100.0 \%$ $[-0.58 ; 1.32]$

\section{Persisting Externalising Problems (3-12 months)}

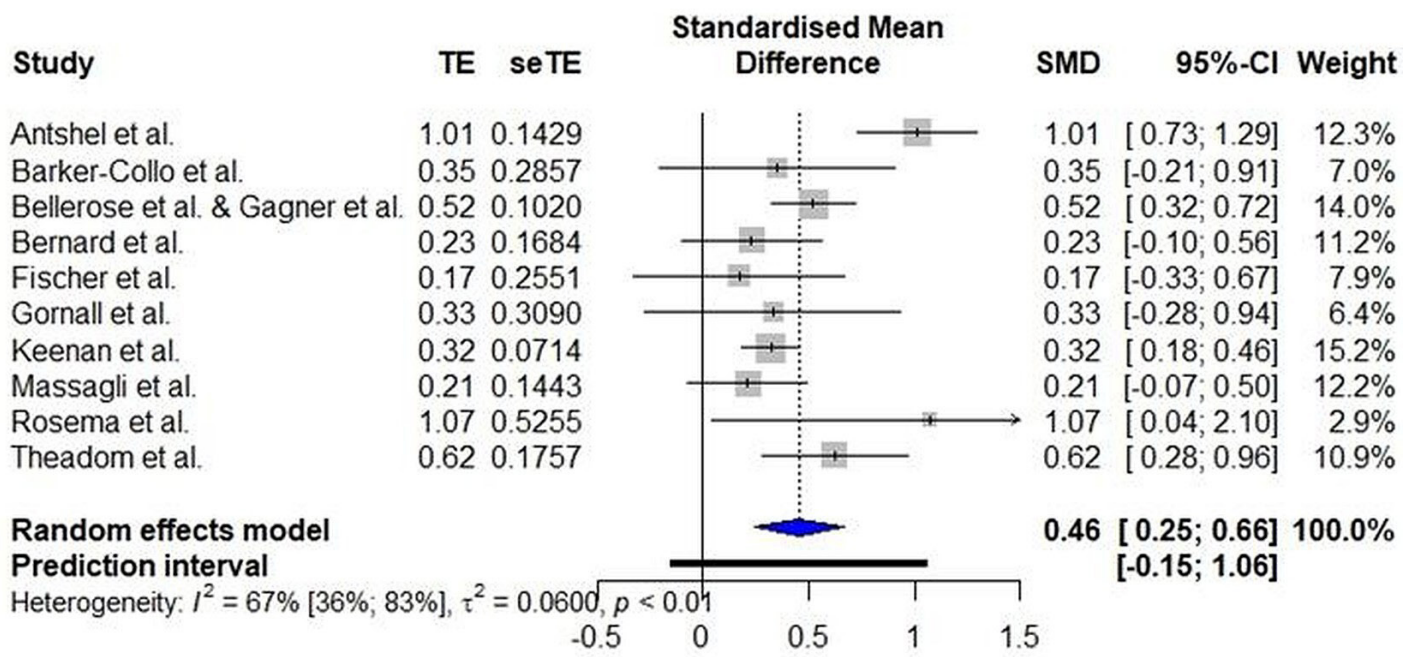

\section{Chronic Externalising Problems (>12 months)}

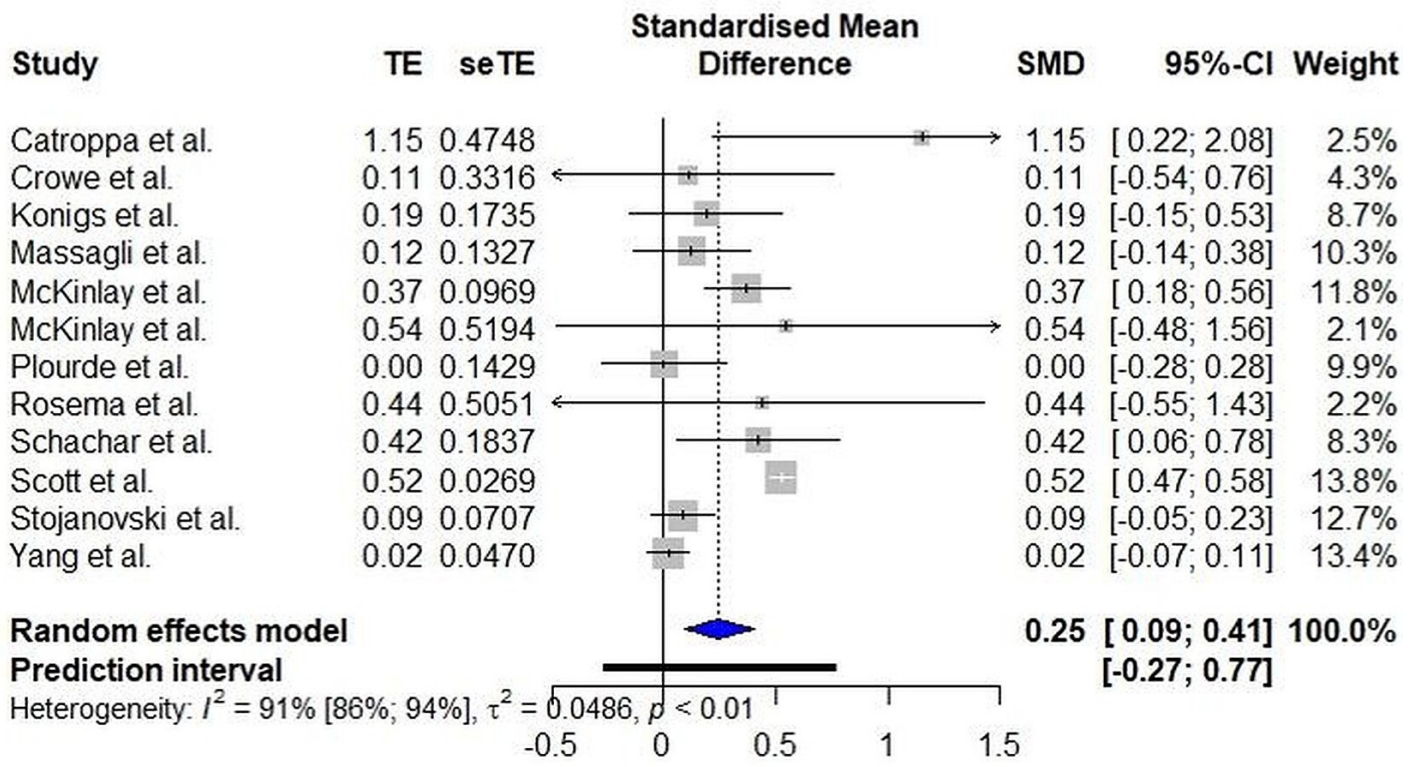

Figure 3 Standard mean difference (SMD), treatment effect (TE) and standard error (seTE) of acute, persisting and chronic externalising problems after paediatric concussion. 


\section{Acute Total Problems ( $<3$ months)}

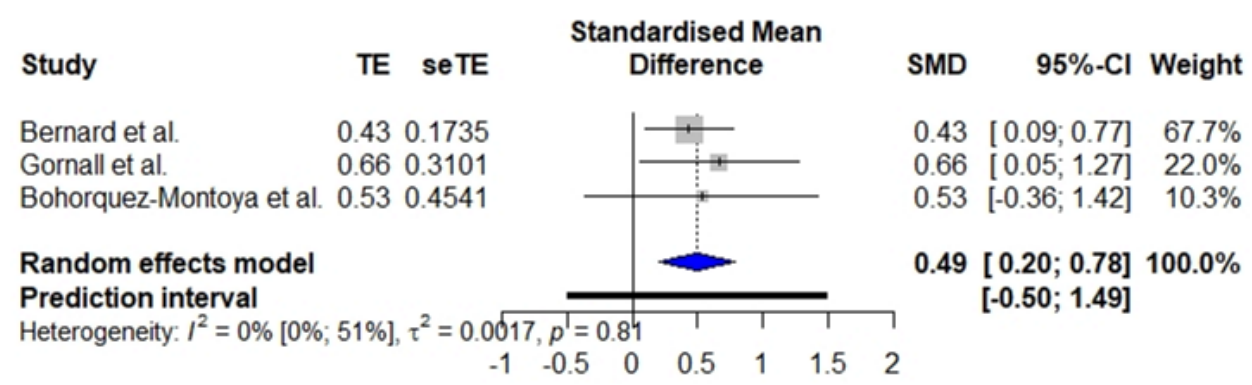

\section{Persisting Total Problems (3-12 months)}

\begin{tabular}{|c|c|c|c|c|c|}
\hline Study & TE seTE & $\begin{array}{l}\text { Standardised Mean } \\
\text { Difference }\end{array}$ & SMD & $95 \%-\mathrm{Cl}$ & Weight \\
\hline Barker-Collo et al. & -0.350 .2857 & \begin{tabular}{l|l}
+ & \\
\end{tabular} & -0.35 & {$[-0.91 ; 0.21]$} & $7.3 \%$ \\
\hline Bernard et al. & 0.340 .1633 & - & 0.34 & {$[0.02 ; 0.66]$} & $12.4 \%$ \\
\hline Brown et al. & 0.140 .0459 & + & 0.14 & {$[0.05 ; 0.23]$} & $18.1 \%$ \\
\hline Gagner et al. & 0.450 .1173 & + & 0.45 & {$[0.22 ; 0.68]$} & $14.9 \%$ \\
\hline Gornall et al. & 0.110 .3086 & + & 0.11 & {$[-0.49 ; 0.71]$} & $6.6 \%$ \\
\hline Keenan et al. & 0.330 .0816 & $\because$ & 0.33 & {$[0.17 ; 0.49]$} & $16.7 \%$ \\
\hline Luis et al. & 0.720 .3184 & - & 0.72 & {$[0.10 ; 1.34]$} & $6.4 \%$ \\
\hline Massagli et al. & 0.380 .0619 & + & 0.38 & {$[0.26 ; 0.50]$} & $17.5 \%$ \\
\hline \multicolumn{2}{|c|}{$\begin{array}{l}\text { Random effects model } \\
\text { Prediction interval }\end{array}$} & & \multirow[t]{2}{*}{0.28} & $\begin{array}{l}{[0.08 ; 0.49]} \\
{[-0.31 ; 0.88]}\end{array}$ & $100.0 \%$ \\
\hline \multirow{2}{*}{\multicolumn{2}{|c|}{ Heterogeneity: $I^{2}=67 \%[30 \% ; 84 \%$}} & .01 & & & \\
\hline & & 0.5 & \multicolumn{2}{|l|}{1.5} & \\
\hline
\end{tabular}

\section{Chronic Total Problems (>12 months)}

\begin{tabular}{|c|c|c|c|c|c|c|}
\hline Study & TE & seTE & $\begin{array}{l}\text { Standardised Mean } \\
\text { Difference }\end{array}$ & SMD & $95 \%-\mathrm{Cl}$ & Weight \\
\hline Brown et al. & 0.10 & 0.0663 & $+:$ & 0.10 & {$[-0.03 ; 0.23]$} & $20.9 \%$ \\
\hline Catroppa et al. & 0.87 & 0.4643 & & 0.87 & {$[-0.04 ; 1.78]$} & $2.2 \%$ \\
\hline Crowe et al. & 0.29 & 0.3316 & & 0.29 & {$[-0.36 ; 0.94]$} & $4.0 \%$ \\
\hline LaliberteDurish et al. & 0.34 & 0.3061 & 4 & 0.34 & {$[-0.26 ; 0.94]$} & $4.6 \%$ \\
\hline Massagli et al., Rockhill et al. & 0.21 & 0.0647 & $\div$ & 0.21 & {$[0.08 ; 0.34]$} & $21.0 \%$ \\
\hline Renstrom et al. & 0.51 & 0.2857 & & 0.51 & {$[-0.05 ; 1.07]$} & $5.1 \%$ \\
\hline Ryan et al. & 0.34 & 0.2245 & $\mp$ & 0.34 & {$[-0.10 ; 0.78]$} & $7.4 \%$ \\
\hline Schachar et al. & 0.32 & 0.1327 & 1 & 0.32 & {$[0.06 ; 0.58]$} & $13.7 \%$ \\
\hline Stojanovski et al. & 0.06 & 0.0646 & 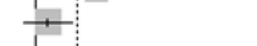 & 0.06 & {$[-0.06 ; 0.19]$} & $21.1 \%$ \\
\hline \multirow{3}{*}{$\begin{array}{l}\text { Random effects model } \\
\text { Prediction interval } \\
\text { Heterogeneity: } I^{2}=21 \%[0 \% ;\end{array}$} & & & & \multirow[t]{3}{*}{0.22} & \multirow{3}{*}{$\begin{array}{l}{[0.09 ; 0.35]} \\
{[-0.15 ; 0.59]}\end{array}$} & $100.0 \%$ \\
\hline & & & 1 & & & \\
\hline & & & 0.5 & & & \\
\hline
\end{tabular}

Figure 4 Standard mean difference (SMD), treatment effect (TE) and standard error (seTE) of acute, persisting and chronic total mental health problems after paediatric concussion.

and $\mathrm{mTBI} /$ complicated $\mathrm{mTBI}$ ) and studies that only reported mental health outcomes for children with delayed recovery were excluded. Reviews, meta-analyses, case studies, conference abstracts, book chapters, commentaries, dissertations, case reports and non-English articles were also excluded.

Eligible studies that included an orthopaedic injury or typically developing control group were included in meta-analyses, while studies that did not include a control group were included in the systematic review only $(n=36)$.

\section{Data classification}

Mental health outcomes were restricted to the broad categories of internalising, externalising and total mental health difficulties, due to the few studies reporting findings at a disorder-specific level. The American Psychological Association endorses the utility of the internalising/externalising framework in research and clinical contexts in their introduction to the Diagnostic and Statistical Manual of Mental Disorders-fifth edition (p.13): 
...clustering of disorders according to what has been termed internalizing and externalizing factors represents an empirically supported framework. Within both the internalizing group (representing disorders with prominent anxiety, depressive, and somatic symptoms) and the externalizing group (representing disorders with prominent impulsive, disruptive conduct, and substance use symptoms), the sharing of genetic and environmental risk factors, as shown by twin studies, likely explains much of the systematic comorbidities seen in both clinical and community samples. ${ }^{33}$

In keeping with the internalising/externalising framework, anxiety, depression, post-traumatic stress and withdrawal were indexed under internalising problems while aggression, conduct problems, attention problems, risk-taking and hyperactivity were classified as externalising problems. ${ }^{33-36}$ Overall mental health was measured by novel psychiatric diagnoses post-injury as well as total problem subscales of validated behavioural and emotional inventories. Pooled effect sizes were calculated for studies reporting results from multiple informants (eg, parent and child self-report) and for different outcomes underlying the same construct.

Evidence suggests that mental health changes with time postconcussion. ${ }^{21}$ However, it is unclear how internalising and externalising difficulties may evolve from very acute stages to longer term impact. We therefore included a wider range of time since injury, to capture and comment on time in the context of recovery. Existing published concussion guidelines and recovery trajectories were used to define time since injury categories. Insufficient data $(n=1)$ were available to analyse outcomes $\leq 1$ month post-injury. Therefore, time points were defined as follows: (1) acute: injury to $<3$ months post-injury, the time frame when most young people recover from concussion ${ }^{8}$; (2) persisting: 3-12 months post-injury, the time frame when a minority of children experience ongoing symptoms ${ }^{837}$; and (3) chronic: $>12$ months post-injury, the time frame when most novel mental health difficulties have emerged, ${ }^{17}$ reflecting the longer term impact on mental health. ${ }^{5}$ Studies were assigned to these categories using the mean time since injury at each assessed time point. Where multiple data points were measured within the same time category, results were pooled into a single effect size.

\section{Quality assessment}

The quality of eligible studies was assessed using the Downs and Black Quality Appraisal Criteria (DBC) which assigns nonintervention studies a maximum of 20 points for the highest quality across five main criteria: (a) external validity, (b) reporting, (c) bias, (d) confounding and (e) power. ${ }^{38}$ The Oxford Centre for Evidence-Based Medicine (OCEBM) 2011 Levels of Evidence were also employed as a measure of evidence strength informed by research design. ${ }^{39}$

\section{Meta-analyses}

Random effects meta-analyses were conducted using meta, ${ }^{40}$ metafor $^{41}$ and dmetar packages in R V.4.0.2. ${ }^{42}$ Means and SDs were used to calculate effect sizes for continuous mental health outcomes, while frequencies of events in cases and controls were used to calculate effect sizes for categorical variables. Where studies did not report adequate data to calculate effect size, the corresponding author was contacted $(n=7)$. Studies that could not be included in the meta-analysis because appropriate data were not provided were included in the systematic review only $(n=2)$.
Effect size was measured using Hedges' g as it adjusts for sample size, thus providing an unbiased estimate of the standardised mean difference. ${ }^{44}$ Consistent with established recommendations for meta-analysis, effect sizes were interpreted as small (0.32), moderate $(0.33-0.55)$ or large $(>0.59) .{ }^{45}$ Heterogeneity was quantified using the $\mathrm{I}^{2}$ statistic which describes the percentage of variability in effect sizes due to heterogeneity rather than chance alone. ${ }^{46}$ Heterogeneity was interpreted as low, or unimportant (0\%-30\%), moderate (30\%-50\%), substantial (50\%-80\%) and considerable (80\%-100\%). ${ }^{47}$ Graphic Display of Heterogeneity (GOSH) plots were generated to explore patterns of heterogeneity, followed by sensitivity analyses. ${ }^{48}$ Heterogeneity was further explored through subgroup analyses and meta-regression where sufficient data were available $(n \geq 10) .{ }^{49}$ Some prespecified analyses were unable to be conducted due to missing data (eg, premorbid mental health status) and were substituted for available alternatives (eg, exclusion of patients with psychiatric history). Influence analyses (eg, 'leave-one-out' approaches) were conducted to ensure that the overall estimate of the metaanalysis was not distorted by outliers or studies exerting high influence on the model. ${ }^{50}$

\section{RESULTS}

\section{Study selection}

The study selection process is illustrated in figure 1. Sixtynine articles representing 60 unique samples satisfied eligibility criteria and were included in the systematic review. Of these, 29 were excluded from the meta-analysis due to the absence of a control group, or because controls did not complete mental health measures, resulting in 40 articles characterising 33 unique studies included in the meta-analytical review. Characteristics and main findings of included studies are displayed in online supplemental table 2 .

\section{Study characteristics}

In total, 89114 children with concussion (60.9\% males) were represented in this meta-analysis. Age at injury ranged from 0 to 18 years, with a weighted grand mean of 11.69 years. Falls $(42.3 \%)$ and sporting injuries $(29.5 \%)$ were the most common injury mechanisms reported, followed by motor vehicle accidents (15.5\%).

One-third of included studies used a prospective longitudinal design $(n=20)$. The remaining studies used prospective cohort $(n=11)$, cross-sectional $(n=9)$, retrospective cohort $(n=9)$, casecontrolled $(n=5)$, inception cohort $(n=3)$, qualitative $(n=2)$, retrospective chart review $(n=1)$ and repeated measures $(n=1)$ designs.

Assessed time points ranged from admission to 20 years postinjury. All studies used age-appropriate, validated measures of mental health $(n=60)$, comprehensive interviews $(n=2)$, formal psychiatric diagnoses $(n=3)$ or engagement with mental health services $(n=3)$ to detect mental health outcomes. The Child Behavior Checklist $(n=19,27.5 \%)$, Behavior Assessment System for Children $(n=7,10.1 \%)$ and Personality Inventory for Children $(n=7,10.1 \%)$ were the most commonly used measures. Other instruments used are listed in online supplemental table 2. Internalising problems were the most frequently assessed mental health outcomes $(n=43)$, followed by overall mental health $(n=32)$ and externalising problems $(n=29)$. Parents were the most frequent respondents $(n=43)$, followed by children $(n=28)$ and teachers $(n=7)$. Fifteen studies included multiple informants (parent and child, $\mathrm{n}=8$; parent and teacher, $\mathrm{n}=6$; parent, child and teacher $\mathrm{n}=1$ ). 
Premorbid mental health status was assessed in 28 studies $(40.6 \%)$, as documented by retrospective ratings of pre-injury behaviour $(n=19)$ or psychiatric diagnoses $(n=9)$. Two studies employed International Classification of Disease Criteria-10, while seven studies did not specify the criteria for pre-injury mental health diagnoses. Thirty-two studies (46.3\%) excluded children either with a history of mental health difficulties or hospitalisation for psychiatric illness. Twenty-one (30.4\%) of the included studies did not account for pre-injury mental health status, nor exclude children with pre-injury mental health diagnoses, while $12(17.4 \%)$ excluded pre-injury mental health diagnoses and included measures of premorbid mental health. Additional study characteristics have been outlined in online supplemental material.

\section{Quality assessment}

All included studies were rated as excellent $(n=41,61.2 \%)$ or good $(n=26,38.8 \%)$ quality on the DBC. Most included studies represented level 3 evidence according to the OCEBM Levels of Evidence $(n=58,82.9 \%)$, with seven inception cohort studies meeting criteria for level $2(10.0 \%)$ and three studies at level $4(4.3 \%)$. Forty-three studies $(61.4 \%)$ recruited participants via consecutive admissions or inception cohort design, suggesting low risk of selection bias among most included studies. Twenty-two studies $(55.0 \%)$ in the meta-analysis recruited controls from the same setting as concussion sample, suggesting risk of selection bias.

Reporting of attrition rates was fair, with 42 studies (60.9\%) describing both proportion and characteristics of participants lost to follow-up. Forty-four studies reported low attrition rates $(<20 \%)$ or adjusted analyses based on confounds related to patients lost to follow-up (63.8\%). Fifty studies (72.5\%) had clear aims and hypotheses. Five (7.2\%) included power calculations. All reported key participant characteristics of patients, and the majority $(n=64,92.8 \%)$ detailed the distribution of key confounders. Of studies included in the meta-analysis, comparability between cases and controls was acceptable, with $34(85.0 \%)$ documenting principal confounds and all studies either demonstrating no significant group differences or making adequate adjustments for confounds.

\section{Systematic review}

The results of included studies are qualitatively synthesised below, corresponding to study research questions.

\section{How common are mental health problems after paediatric concussion?}

Twenty-nine articles addressed this question. Studies consistently reported that a minority of children experienced clinically significant levels of internalising, externalising and total mental health problems after concussion ${ }^{16-18} 28{ }^{51-59}$ : $19.3 \%-40.0 \%$ of whom reported no pre-injury mental health symptoms and 50.0\%$60.0 \%$ reported a history of elevated pre-injury mental health symptoms $^{56-58}$ or diagnoses. ${ }^{17} 18$ Twenty-nine per cent of children with pre-injury mental health diagnoses received a novel mental health diagnosis post-concussion. ${ }^{17}$ Internalising, externalising and total mental health problems were reported within the normal range in most studies. ${ }^{21} 60-64$ One study reported mean behaviour problems in the clinical range 3 months postinjury with resolution by 6 months. ${ }^{61}$

The prevalence of clinically significant internalising problems ranged from $3.0 \%$ to $36.7 \%,{ }^{5465-68}$ with one study reporting $40 \%$ of children demonstrated subclinical anxiety following concussion. ${ }^{69}$ Although parents reported their child's inability to control anger among the most distressing symptoms, ${ }^{70}$ just one study reported the proportion of clinical externalising problems following concussion, at 20.0\% 16 years post-injury. ${ }^{71}$ Results from a population-based study by McKinlay et al found that preschool children who attended hospital for concussion were three to four times more likely to show symptoms of attentiondeficit/hyperactivity disorder, conduct disorder, oppositional defiant disorder, substance abuse and mood disorder during early adolescence, compared with typically developing controls and children who presented to outpatient services after concussion. $^{28} 51$

What factors predict mental health following paediatric concussion? Studies reported several factors influenced mental health after paediatric concussion $(n=28)$. Pre-injury mental health was consistently identified as a strong predictor of post-injury mental health problems. ${ }^{16} 1757667273$ Five studies systematically examined this relationship with regression analyses and demonstrated that psychiatric history explained between $38.4 \%$ and $65.0 \%$ of variance in mental health problems post-concussion. ${ }^{16} 17667374$ Age and sex were inconsistent predictors of mental illness. Internalising vulnerabilities were associated with female sex ${ }^{2122} 5275-77$ and older age at injury. ${ }^{1721606178}$ Females appeared at greater risk of developing novel mental health difficulties after concussion, ${ }^{21527576}$ but this finding was not universal. ${ }^{1755}$ Males with a psychiatric history were at increased risk of developing further psychiatric illness after concussion. ${ }^{17}$ Younger age at injury was associated with more externalising problems ${ }^{17} 22$ and post-traumatic stress symptoms ${ }^{76}$ however some studies found no association between age and mental health or psychosocial functioning. ${ }^{65}{ }^{69}$ While several studies indicated a role for family factors, ${ }^{17185257687980}$ only five studies explored family characteristics post-concussion. ${ }^{1622728081}$ Results showed that family functioning, parent mental health, parent distress and family characteristics (eg, family living arrangement, parent education) were associated with externalising problems 6 months after concussion. ${ }^{1622728081}$ In contrast, family characteristics did not predict internalising problems above and beyond participant characteristics. ${ }^{16}$

Two studies explored protective factors associated with better mental health outcomes, identifying positive family environment, cultural and psychological resilience as associated with better quality of life and lower levels of depression, anxiety, adjustment and attention-deficit/hyperactivity symptoms. ${ }^{22} 75$

How does mental health interact with recovery from concussion? Few studies directly examined the interaction between mental health and PCS $(n=11)$. Internalising symptoms significantly predicted acute and post-acute PCS. ${ }^{5} 7582$ Higher premorbid PCS predicted increased psychological difficulties over time. ${ }^{5273}$ Nonetheless, even when taking pre-injury data into account, considerable variation in recovery trajectories exists. ${ }^{56}$

How does mental health change with time post-injury?

In the nine studies addressing this issue, mental health symptom profile tended to remain stable over time, ${ }^{69}$ with most novel psychiatric diagnoses developing in the first-year postconcussion. ${ }^{17}$ Significant improvements in mental health emerged between 3 and 6 months post-injury. ${ }^{21} 7283$ Among infants and toddlers, social and emotional behaviour worsened over time, ${ }^{84}$ with significantly higher rates of conduct, oppositional defiant, 
attention-deficit/hyperactivity, substance use and mood disorder symptoms evident several years post-concussion. ${ }^{28}$

\section{Meta-analyses}

We then conducted meta-analyses to determine if children experienced elevated mental health difficulties after concussion compared with controls.

\section{Internalising problems}

Significant, moderate effects were observed in the concussion group compared with controls across acute $(t=5.12, g=0.45$, $95 \%$ CI $(0.17$ to 0.74$), \mathrm{p}=0.01)$ persisting $(\mathrm{t}=4.26, \mathrm{~g}=0.42$, $95 \% \mathrm{CI}(0.20$ to 0.63$), \mathrm{p}=0.001)$ and chronic $(\mathrm{t}=2.88, \mathrm{~g}=0.41$, $95 \%$ CI $(0.10$ to 0.72$), p=0.01)$ outcomes (see figure 2 ). At each time point, there was a large prediction interval and low to considerable heterogeneity (acute: $\mathrm{I}^{2}=0.0 \%, 95 \% \mathrm{CI}(0.0 \%$ to $76.8 \%), Q(3)=1.98, \mathrm{p}=0.58$; persisting: $\mathrm{I}^{2}=51.0 \%, 95 \% \mathrm{CI}$ (5.3\% to $74.7 \%), Q(11)=22.46, p=0.02$; chronic: $\mathrm{I}^{2}=49.3 \%$, $95 \% \mathrm{CI}(6.2 \%$ to $72.6 \%), \mathrm{Q}(13)=25.67, \mathrm{p}=0.02)$. GOSH diagnostics identified outliers contributing to cluster imbalance in both persisting $(n=2)$ and chronic models $(n=4)$. Pooled estimates remained significant omitting these outliers and heterogeneity was low to moderate (persisting: $\mathrm{g}=0.46,95 \% \mathrm{CI}(0.32$ to 0.59$), \mathrm{p}<0.0001, \mathrm{I}^{2}=36.1 \%$; chronic: $\mathrm{g}=0.27,95 \% \mathrm{CI}(0.11$ to 0.42$), p=0.003, I^{2}=28.9 \%$; online supplemental figure 1 ). Leave-one-out analyses revealed that all pooled estimates after excluding one study at a time fell within the 95\% CI of the overall estimate. Funnel plots and Egger's test showed evidence of publication bias in chronic internalising outcomes $(p=0.003)$. Nonetheless, p-curve analyses supported evidential value suggesting that a true effect size underlies presented findings and that results are not the product of publication bias and $\mathrm{p}$-hacking alone.

\section{Externalising problems}

Significant, moderate effect sizes were observed across acute $(\mathrm{t}=5.77, \mathrm{p}=0.03, \mathrm{~g}=0.37,95 \% \mathrm{CI}(0.09$ to 0.65$))$ and persisting $(\mathrm{t}=4.94, \mathrm{p}<0.001, \mathrm{~g}=0.46,95 \% \mathrm{CI}(0.25$ to 0.66$))$ time points, decreasing to a small effect in the chronic model $(t=3.46$, $\mathrm{p}=0.005, \mathrm{~g}=0.25,95 \% \mathrm{CI}(0.09$ to 0.41$)$; see figure 3$)$. Heterogeneity was low to moderate and non-significant for acute externalising problems $\left(\mathrm{I}^{2}=0.00 \%, 95 \% \mathrm{CI}(0.00 \%\right.$ to $50.5 \%)$, $\mathrm{Q}(2)=0.42, \mathrm{p}=0.81)$ but substantial and significant among studies investigating persisting $\left(\mathrm{I}^{2}=66.9 \%, 95 \% \mathrm{CI}(35.5 \%\right.$ to $83.0 \%), \mathrm{Q}(9)=27.18, \mathrm{p}=0.001)$ and chronic effects $\left(\mathrm{I}^{2}=90.7 \%\right.$, $95 \%$ CI $(85.7 \%$ to $94.0 \%), \mathrm{Q}(11)=118.32, \mathrm{p}<0.0001)$. GOSH diagnostics identified outliers contributing to cluster imbalance in both persisting $(n=3)$ and chronic models $(n=3)$. We reperformed the meta-analyses omitting outliers and the pooled estimates remained significant with low heterogeneity $(\mathrm{g}=0.38$, $95 \%$ CI $(0.21$ to 0.54$), \mathrm{p}=0.001, \mathrm{I}^{2}=8.4 \% ; \mathrm{g}=0.19,95 \% \mathrm{CI}$ (0.07 to 0.32 ), $\mathrm{p}=0.007, \mathrm{I}^{2}=18.8 \%$; online supplemental figure $2)$. Leave-one-out analyses revealed that all pooled estimates after excluding one study at a time fell within the 95\% CI of the overall estimate. No evidence of publication bias was identified on funnel plots or Egger's test. Evidential values supported a true effect size underlying present results.

\section{Total problems}

Significant, small to moderate effects were observed across acute $(\mathrm{t}=7.34, \mathrm{p}=0.02, \mathrm{~g}=0.49,95 \% \mathrm{CI}(0.20$ to 0.78$))$, persisting $(\mathrm{t}=3.24, \mathrm{p}=0.01, \mathrm{~g}=0.28,95 \% \mathrm{CI}(0.08$ to 0.49$))$ and chronic total mental health problems $(t=3.91, p=0.005, g=0.22,95 \% \mathrm{CI}$
(0.09 to 0.35$)$ ), with effect size decreasing over time (see figure 4 ). Heterogeneity was low to moderate and non-significant across the acute $\left(\mathrm{I}^{2}=0.00 \%, 95 \% \mathrm{CI}(0.00 \%\right.$ to $51.4 \%), \mathrm{Q}(2)=0.43$, $\mathrm{p}=0.81)$ and chronic time points $\left(\mathrm{I}^{2}=20.7 \%, 95 \% \mathrm{CI}(0.00 \%\right.$ to $62.0 \%), \mathrm{Q}(8)=10.09, \mathrm{p}=0.26)$, but moderate to high in the persisting model $\left(\mathrm{I}^{2}=66.7 \%\right.$, 95\% CI $(29.6 \%$ to $84.3 \%)$, $\mathrm{Q}(7)=21.03, \mathrm{p}=0.004)$. GOSH diagnostics identified outliers contributing to cluster imbalance in both persisting $(n=2)$ and chronic models $(n=1)$. We reperformed meta-analyses omitting outliers, and pooled estimates remained significant with low heterogeneity $(\mathrm{g}=0.38,95 \% \mathrm{CI}(0.26$ to 0.49$), \mathrm{p}=0.0004$, $\mathrm{I}^{2}=0.0 \% ; \mathrm{g}=0.18,95 \% \mathrm{CI}(0.08$ to 0.29$), \mathrm{p}=0.004, \mathrm{I}^{2}=9.4 \%$; online supplemental figure 3). Leave-one-out analyses revealed that all pooled estimates after excluding one study at a time fell within the $95 \%$ CI of the overall estimate. Chronic, but not persisting, outcomes showed evidence of publication bias on funnel plot and Egger's test $(p=0.02)$; however, evidential values supported a true underlying effect.

\section{Additional analyses}

Meta-analyses stratified by age and sex were not possible due to the lack of age-specific and sex-specific data reported in the included studies. Meta-regressions evaluating the impact of sex (\% males) and age (mean age) on mental health were, therefore, conducted. Variability in persisting internalising and externalising outcomes was not explained by any of the demographic or methodological factors examined (see online supplemental tables 2 and 3). Chronic internalising problems significantly differed by OCEBM Level of Evidence, with level 3 studies showing the greatest effect size $(g=0.51)$, followed by levels 4 $(g=0.38)$ and $2(g=0.10)$. Sex and DBC quality rating accounted for $7.78 \%$ and $5.63 \%$ of variance, respectively, but were nonsignificant predictors overall. In the chronic externalising model, mean age accounted for $5.00 \%$ of variance, but was a non-significant predictor overall. As with persisting outcomes, no other factors significantly accounted for heterogeneity at the chronic time point. Insufficient studies were available to conduct subgroup analyses or meta-regressions on total behaviour problems. Online supplemental figures 4-7 present forest plots by design and respondent.

\section{DISCUSSION}

The aim of our paper was to systematically investigate mental health outcomes following paediatric concussion while controlling for key methodological differences that have limited synthesis of paediatric concussion research. Our results revealed that mental health is highly relevant to concussion recovery. Both the qualitative synthesis and meta-analysis demonstrated that, even among studies with homogeneous designs, samples and concussion definitions, mental health outcomes can vary widely. This indicates that children respond to concussion in diverse ways. While mental health generally improves with time post-injury, a minority of children experience clinically significant and persisting mental health symptoms that warrant clinical attention. Post-traumatic stress and depressive symptoms in particular have been associated with increased risk for adverse outcomes after concussion. ${ }^{54} 85$ Evidence suggests that pre-existing mental health symptoms, family anxiety, the psychosocial impact of post-concussion symptoms and reduced activity may contribute to ongoing mental health symptoms in this group. ${ }^{16175766727386-88}$ Despite this chronic vulnerability, only a small proportion of children 
access mental healthcare after concussion, ${ }^{5259}$ highlighting this as a missed opportunity for early intervention.

In contrast to recovery from PCS more generally, ${ }^{8}$ few studies reported significant improvements in mental health symptoms by 1 month post-injury, ${ }^{83}$ suggesting that mental health symptoms may take longer to resolve than other post-concussion symptoms. This suggests that concussion is an important factor precipitating subsequent mental health symptoms, which may be maintained by biopsychosocial factors related to concussion (eg, fatigue) and context (eg, worry about repeat injury). Of note, the most commonly employed measures of PCS include symptoms that overlap considerably with mental health (eg, emotional lability, irritability, nervousness, sadness), leading to difficulties differentiating PCS and mental health symptoms. ${ }^{89}$ Despite this confound, few studies commented on the potential overlap in measurement of these two dimensions. This relationship may be driven by a high level of symptom overlap between PCS and mental health difficulties, or by a feedback loop where persisting PCS precipitate acute stress which then exacerbates mental health symptoms, which further impacts PCS.

The less competitive nature of sport among younger children contributes difficulties differentiating sport from nonsport concussion in paediatrics. Therefore, little research has been conducted on sport concussion in children under 13 years, ${ }^{9}$ despite a significant proportion of paediatric concussions sustained during sport. ${ }^{90}$ Sport-concussion research is important given the increased risk of repeated concussions, ${ }^{9}$ which has been associated with mental health difficulties and delayed recovery. ${ }^{414}$ Results from this review highlight that mental health outcomes from sport ${ }^{53} 778291$ and non-sport concussion are broadly consistent, despite greater representation of older children and adolescents in sport concussion research. While clinical recommendations for non-sportrelated concussions are lacking, these findings highlight that, alongside clinical judgement, sport-related concussion guidelines are likely to be highly relevant to the assessment and management of non-sport concussion.

Current evidence establishes pre-injury mental health difficulties as the strongest and most consistent predictor of post-concussion mental health outcomes; however, novel diagnoses occur in approximately one-third of young people after concussion. ${ }^{17}$ Female sex was associated with increased internalising problems after concussion, however, this vulnerability is also evident in the general population and is unlikely to be concussion specific. Age was an inconsistent predictor of mental health outcomes. Interaction effects between age and sex are a potential explanation for this variability with internalising problems increasing with age among females. ${ }^{21}$ Given that female sex and older adolescence have also been identified as risk factors for delayed recovery following concussion, ${ }^{4}$ present results may reflect vulnerability to adverse outcomes from concussion more broadly, the emergence of mental health difficulties observed among females during adolescence, or an interaction between these factors and their psychosocial context.

Infants and toddlers may also experience social and emotional difficulties that worsen over time, ${ }^{84}$ with significantly higher rates of conduct, oppositional defiant, attention-deficit/ hyperactivity, substance use and mood disorder symptoms evident several years post-concussion. ${ }^{28}$ This finding might be explained by the fact that younger children are less likely to have existing mental health diagnoses, reflect developmentally specific neurobehavioural outcomes, family anxiety, incidental reinforcement of maladaptive coping (eg, reassurance seeking, separation anxiety) or a combination of these factors. Further research is needed to explore the relationship between concussion, mental health and child development in greater detail.

Psychological resilience and family characteristics emerged as promising, but largely unexplored, predictors of postconcussion mental health outcomes. Given these domains offer the potential for early intervention, further exploration of these factors may provide opportunities to improve mental health outcomes.

Consistent with reviews of elite collegiate athletes, ${ }^{92}$ our results support an association between concussion and acute internalising mental health symptoms. While depressive symptoms in elite collegiate athletes resolve within 1 month postconcussion, ${ }^{92}$ our review suggests that both internalising and externalising problems persist longer in children and adolescents. Such long-term mental health problems have also been highlighted in a narrative review of suicidality after concussion and mTBI among children, adults and military personnel, showing a twofold greater risk of suicide compared with peers. $^{93}$ Our review extends our understanding of mental health consequences of concussion to children and adolescents, who may be at greatest risk of adverse outcomes. ${ }^{9}$

This systematic review and meta-analysis included studies employing a variety of designs, providing an opportunity to explore the impact of research design on study results. Prospective cohort and longitudinal prospective cohorts generally found larger effects than cross-sectional, case-control, retrospective cohort and inception cohort designs. This may reflect bias in reporting positive results or strengths of prospective cohort designs, including accuracy of data collection regarding exposures, confounders and timing of concussion. Population studies often revealed long-term impacts that were not evident in many retrospective designs. ${ }^{596594-96}$ Consistent with population data, 2728517897 several prospective longitudinal cohort studies reported increased mental health difficulties several years post-concussion. ${ }^{15165762717598}$

\section{Clinical implications}

Results from our review highlight the importance of considering mental health as a predisposing, exacerbating and protective factor for concussion recovery. Findings that PCS subtypes are relatively stable over time since injury ${ }^{1769}$ highlight the potential and importance of early intervention. When making recommendations for concussion management, clinicians should consider potential mental health consequences, particularly where premorbid mental health difficulties are present.

Previous management approaches, such as prolonged rest, have been associated with poor mental health and adverse outcomes after concussion. ${ }^{878}$ Current clinical guidelines for recovery support a systematic and graduated return to exercise, school and sport. ${ }^{99} 100$ The CISG Berlin Consensus Statement outlines the importance of applying this protocol alongside clinical judgement, on an individual basis. ${ }^{9}$ The statement further highlights the utility of structured interventions, such as graded exercise ${ }^{101}$ and physiotherapy tailored to individual symptoms. ${ }^{102} 103$ Less is known about treatment of mental health symptoms, with initial promise shown with the use of cognitive-behavioural paradigms, ${ }^{104}$ either alone or as part of collaborative care models. The trajectories of PCS and mental health symptoms are closely intertwined among children with 
delayed recovery, ${ }^{105}$ further demonstrating mental health as an important target for intervention.

Since the majority of children and adolescents with clinical mental health difficulties do not access recommended care, ${ }^{106}$ incorporating mental health risk into post-injury management represents an avenue to engage young people with mental health services to either prevent unnecessary problems emerging or to treat premorbid issues.

The presence of psychological distress is associated with twice the healthcare costs after paediatric concussion, ${ }^{107}$ further highlighting the importance of early intervention. When assessing persisting post-concussion symptoms, clinicians should be cognisant of the potential overlap between PCS and mental health symptoms. As a first step, mental health should be evaluated as part of standard acute concussion assessment and management. This may encourage families to monitor and seek support for emerging or exacerbated emotional and psychological symptoms. Among children with delayed recovery, parent anxiety contributes additional family burden following childhood concussion, indicating a need for management strategies to consider the implications to the entire family. ${ }^{86}$

Anxiety and depression significantly mediated the relationship between resilience and post-concussion symptoms, ${ }^{108}$ suggesting that mental health has an important impact on recovery from concussion, even accounting for individual characteristics. While children provided different reasons for their emotional distress (eg, inability to play sport, others' lack of understanding about concussion), they consistently reported frustration, depression, anxiety, irritability and feelings of isolation associated with their injury. ${ }^{91}$ Considered together, current evidence supports the use of a biopsychosocial model for understanding the factors contributing to the development and maintenance of mental health difficulties post-concussion on an individual basis.

\section{Strengths and limitations}

This systematic review extends past literature by focusing specifically on mental health after concussion in a paediatric population and is among the first to perform a meta-analysis. Major strengths are precise inclusion criteria, thorough search strategy and robust evaluation. Nonetheless, several limitations need to be acknowledged. First, we excluded unpublished and non-English language articles, which may subject findings to publication and language bias. Although risk of publication bias was identified for some comparisons, further analyses supported the presence of a true effect underlying results. This systematic review and meta-analysis included studies employing a variety of designs, assessment tools and respondents. While this can increase heterogeneity, it also provides an important opportunity to comment on the impact of research design on mental health outcomes and focus of past research. To balance these considerations, we restricted inclusion to studies that measured mental health prospectively, regardless of how other unrelated measures were obtained. Considering the relatively small number of studies and the results of subgroup analyses conducted, the impact on the overall conclusions is minimal.

\section{Limitations of the literature}

At a study level, only four studies explored mental health outcomes within the first 3 months of concussion. Forty-one studies (59.4\%) addressed mental health as the primary

\section{What is already known?}

Concussion is a common injury among children and adolescents.

- While some studies report significant mental health problems after childhood concussion, other studies report no such finding.

- Several post-concussion symptoms overlap with mental health symptoms, however, the relationship between delayed recovery and mental health remains poorly understood.

\section{What are the new findings?}

- Mental health is highly relevant to concussion recovery, although the ways children respond to concussion are diverse.

- A minority of children experience clinically significant mental health symptoms that warrant clinical attention, particularly in the presence of post-traumatic stress symptoms and depression.

- Current evidence establishes pre-injury mental health difficulties as the strongest and most consistent predictor of paediatric post-concussion mental health outcomes, however, novel diagnoses occur in approximately one-third of young people after concussion.

- Psychological resilience and family characteristics are promising, but largely unexplored, predictors of postconcussion mental health outcomes.

- In contrast to recovery from post-concussion symptoms more generally, mental health symptoms may take longer than 1 month to resolve.

- Current evidence supports the use of a biopsychosocial model for understanding the factors contributing to the development and maintenance of mental health difficulties post-concussion on an individual basis.

\section{How might it impact on clinical practice in the future?}

- Mental health should be evaluated as part of standard paediatric concussion assessment and management in the context of biopsychosocial factors.

- Mental health should be considered a potential predisposing, exacerbating and protective factor for paediatric concussion recovery.

- When making recommendations for concussion management in children, clinicians should consider potential mental health consequences, particularly where premorbid mental health difficulties are present.

- Incorporating mental health risk into post-injury management represents an avenue to engage young people with mental health services to either prevent unnecessary problems emerging or to treat premorbid issues.

- When assessing persisting post-concussive symptoms in children, clinicians should be cognisant of the potential overlap between post-concussive symptoms and mental health symptoms. 
outcome. In the remaining studies, mental health was a secondary consideration to cognition $(\mathrm{n}=11)$, physiology $(n=10)$, sleep $(n=3)$ or quality of life related $(n=4)$. Only two studies separated mental health data by sex and age group, highlighting significant gaps in past research and limiting the generalisability of acute findings.

Pre-injury mental health was reported in less than half of the included studies. While most of these studies employed a validated measure of pre-injury mental health, few reported the criteria for formal diagnosis. Most studies relied on normed and validated parent or self-report mental health measures. While child respondents generally reported more mental health difficulties than parents and diagnoses reported in medical records, results were broadly consistent across studies. Studies including multiple respondents showed lower variability than single respondents highlighting valuable increase in precision. Finally, although 69 studies were identified as eligible for the systematic review, nearly half of these were excluded from the metaanalysis due to the absence of a control group.

\section{Future directions}

Future studies seeking to address design limitations should aim to assess pre-injury mental health, incorporate clinical assessment and include multiple respondents with a robust, clearly defined measure of pre-injury mental health. Reporting age-specific and sex-specific results will clarify the relationship to mental health outcomes highlighted in this review. Methodology would be strengthened by including a control group, reporting power analyses and continuing to minimise bias wherever possible. The complex relationship between mental health and concussion recovery requires further study, with particular attention focused on the role that personality, resilience, family, developmental and environmental factors may play in mental health after paediatric concussion. In-depth clinical interviews and qualitative research designs, largely lacking to date, are needed to extend these quantitative findings and draw out these complex relationships. To date, many studies have excluded children with a history of mental health diagnoses. Given the community prevalence of mental illness among children and adolescents and the potential role that mental health may play in recovery from concussion, this represents an important limitation, which likely skews our current understanding of concussion recovery and its underlying mechanisms. Mental health was predominantly reported by parents, with only a quarter of studies including multiple informants. Given that parents' understanding of concussion may influence their perceptions and attributions of post-injury mental health difficulties, ${ }^{80}$ future research should obtain a range of perspectives.

\section{CONCLUSIONS}

Our study revealed that children experience elevated mental health difficulties after concussion which can persist several years postinjury. Assessment, prevention and intervention of mental health difficulties after concussion should be integrated into standard concussion management. A range of biopsychosocial factors need to be considered to clarify the nature of post-concussion recovery on an individual basis. Further research is needed to illuminate the mechanisms underlying observed relationships between mental health, PCS and psychosocial factors.

\section{Twitter Vicki Anderson @VickiAn28323584}

Acknowledgements The authors would like to thank all researchers who provided additional data for inclusion in this meta-analysis and the librarian, Poh Chua, from the Royal Children's Hospital for her assistance in designing the search strategy used in this review.
Contributors AG designed the search strategy, conducted the systematic searches, completed title, abstract and full-text screening, completed quality analysis, conducted meta-analyses and qualitative synthesis, and drafted the manuscript. TM co-screened title and abstracts for this review. XL co-conducted the full-text review. MT completed quality analysis consensus and critically revised the manuscript for important intellectual content. VA contributed to the conception of this study and critically revised the manuscript for important intellectual content. All authors approved the final manuscript as submitted and agree to be accountable for all aspects of the work.

Funding This research was supported by the Victorian Government Operational Infrastructure Scheme. MT is funded by a research grant from the Royal Children's Hospital Foundation. VA is funded by an Australian National Health and Medical Research Council Senior Practitioner Fellowship. This research was conducted as part of AG's PhD research which is supported by the Australian Government Research Training Scheme.

\section{Competing interests None declared.}

\section{Patient consent for publication Not required.}

Provenance and peer review Not commissioned; externally peer reviewed.

Supplemental material This content has been supplied by the author(s). It has not been vetted by BMJ Publishing Group Limited (BMJ) and may not have been peer-reviewed. Any opinions or recommendations discussed are solely those of the author(s) and are not endorsed by BMJ. BMJ disclaims all liability and responsibility arising from any reliance placed on the content. Where the content includes any translated material, BMJ does not warrant the accuracy and reliability of the translations (including but not limited to local regulations, clinical guidelines, terminology, drug names and drug dosages), and is not responsible for any error and/or omissions arising from translation and adaptation or otherwise.

\section{ORCID iDs}

Alice Gornall http://orcid.org/0000-0002-0406-3266

Michael Takagi http://orcid.org/0000-0003-2903-7598

Vicki Anderson http://orcid.org/0000-0001-5233-3147

\section{REFERENCES}

1 Hux K, Dymacek R, Childers C. Possible brain injury events and symptoms in elementary school children. Brain Inj 2013;27:1348-55.

2 Makdissi M, Schneider KJ, Feddermann-Demont N, et al. Approach to investigation and treatment of persistent symptoms following sport-related concussion: a systematic review. Br J Sports Med 2017:51:958-68.

3 Barlow KM. Postconcussion syndrome: a review. J Child Neurol 2016;31:57-67.

4 Zemek R, Barrowman N, Freedman SB, et al. Clinical risk score for persistent Postconcussion symptoms among children with acute concussion in the ED. JAMA 2016:315:1014-25.

5 Anderson V, Davis GA, Takagi M, et al. Trajectories and predictors of ClinicianDetermined recovery after child concussion. J Neurotrauma 2020;37:1392-400.

6 Willer B, Dumas J, Hutson A, et al. A population based investigation of head injuries and symptoms of concussion of children and adolescents in schools. Inj Prev 2004:10:144-8

7 Babl FE, Pfeiffer H, Dalziel SR, et al. Paediatric intentional head injuries in the emergency department: a multicentre prospective cohort study. Emerg Med Australas 2019;31:546-54

8 Davis $\mathrm{GA}$, Anderson $\mathrm{V}$, Babl FE, et al. What is the difference in concussion management in children as compared with adults? A systematic review. Br J Sports Med 2017;51:949-57.

$9 \mathrm{McC}$ rory $\mathrm{P}$, Meeuwisse W, Dvořák J, et al. Consensus statement on concussion in sport-the $5^{\text {th }}$ international conference on concussion in sport held in Berlin, October 2016. Br J Sports Med 2017:51:838-47.

10 Julien J, Tinawi S, Anderson K, et al. Highlighting the differences in post-traumatic symptoms between patients with complicated and uncomplicated mild traumatic brain injury and injured controls. Brain Inj 2017;31:1846-55.

11 Iverson GL, Lange RT. Examination of "postconcussion-like" symptoms in a healthy sample. App/ Neuropsychol 2003;10:137-44.

12 Hou R, Moss-Morris R, Peveler $R$, et al. When a minor head injury results in enduring symptoms: a prospective investigation of risk factors for postconcussional syndrome after mild traumatic brain injury. J Neurol Neurosurg Psychiatry 2012;83:217-23.

13 Iverson GL, Gardner AJ, Terry DP, et al. Predictors of clinical recovery from concussion: a systematic review. Br J Sports Med 2017;51:941-8.

14 Emery CA, Barlow KM, Brooks BL, et al. A systematic review of psychiatric, psychological, and behavioural outcomes following mild traumatic brain injury in children and adolescents. Can J Psychiatry 2016:61:259-69.

15 Catroppa C, Godfrey C, Rosenfeld JV, et al. Functional recovery ten years after pediatric traumatic brain injury: outcomes and predictors. J Neurotrauma 2012:29:2539-47.

16 Gagner C, Landry-Roy C, Bernier A, et al. Behavioral consequences of mild traumatic brain injury in preschoolers. Psychol Med 2018;48:1551-9. 
17 Massagli TL, Fann JR, Burington BE. Psychiatric illness after mild traumatic brain injury in children. Arch Phys Med Rehab 2004;85:1428-34.

18 Luis CA, Mittenberg W. Mood and anxiety disorders following pediatric traumatic brain injury: a prospective study. J Clin Exp Neuropsychol 2002;24:270-9.

19 Ryan NP, van Bijnen L, Catroppa C, et al. Longitudinal outcome and recovery of social problems after pediatric traumatic brain injury (TBI): contribution of brain insult and family environment. Int J Dev Neurosci 2016;49:23-30.

20 Wetherington CE, Hooper SR, Keenan HT, et al. Parent ratings of behavioral functioning after traumatic brain injury in very young children. J Pediatr Psychol 2010;35:662-71.

21 Gornall A, Takagi M, Clarke C, et al. Behavioral and emotional difficulties after pediatric concussion. J Neurotrauma 2020;37:163-9.

22 Keenan HT, Clark AE, Holubkov R, et al. Psychosocial and executive function recovery trajectories one year after pediatric traumatic brain injury: the influence of age and injury severity. J Neurotrauma 2018;35:286-96.

23 Laliberté Durish C, Pereverseff RS, Yeates KO. Depression and depressive symptoms in pediatric traumatic brain injury: a scoping review. J Head Trauma Rehabil 2018;33:E18-30.

24 Yeates KO, Taylor HG, Rusin J, et al. Longitudinal trajectories of postconcussive symptoms in children with mild traumatic brain injuries and their relationship to acute clinical status. Pediatrics 2009;123:735-43.

25 Fay TB, Yeates KO, Taylor HG, et al. Cognitive reserve as a moderator of postconcussive symptoms in children with complicated and uncomplicated mild traumatic brain injury. J Int Neuropsychol Soc 2010;16:94-105.

26 Papoutsis J, Stargatt R, Catroppa C. Long-term executive functioning outcomes for complicated and uncomplicated mild traumatic brain injury sustained in early childhood. Dev Neuropsychol 2014;39:638-45.

27 McKinlay A, Dalrymple-Alford JC, Horwood LJ, et al. Long term psychosocial outcomes after mild head injury in early childhood. I Neurol Neurosurg Psychiatry 2002;73:281-8

28 McKinlay A, Grace R, Horwood J, et al. Adolescent psychiatric symptoms following preschool childhood mild traumatic brain injury: evidence from a birth cohort. J Head Trauma Rehabil 2009:24:221-7.

29 Hessen E, Nestvold K. Indicators of complicated mild TBI predict MMPI-2 scores after 23 years. Brain Inj 2009;23:234-42.

30 Olsson KA, Lloyd OT, Lebrocque RM, et al. Predictors of child post-concussion symptoms at 6 and 18 months following mild traumatic brain injury. Brain Inj 2013;27:145-57.

31 Si B, Dumkrieger G, Wu T, et al. Sub-classifying patients with mild traumatic brain injury: a clustering approach based on baseline clinical characteristics and 90-day and 180-day outcomes. PLoS One 2018;13:e0198741.

32 Covidence. Covidence systematic review software. Veritas health innovation: Melbourne, Australia, 2018. Available: www.covidence.org

33 American Psychiatric Association. Diagnostic and statistical manual of mental disorders (DSM-5®). American Psychiatric Pub, 2013.

34 Achenbach T. Manual for the child behavior Checklist/4-18 and 1991 profile. University of Vermont, Department of Psychiatry, 1991.

35 Reynolds CR KR. Behavior assessment system for children. 3 edn. Bloomington, MN: Pearson, 2015.

36 Goodman R. Psychometric properties of the strengths and difficulties questionnaire J Am Acad Child Adolesc Psychiatry 2001;40:1337-45.

37 Ledoux A-A, Tang K, Yeates KO, et al. Natural progression of symptom change and recovery from concussion in a pediatric population. JAMA Pediatr 2019;173:e183 820-e.

38 Downs SH, Black N. The feasibility of creating a checklist for the assessment of the methodological quality both of randomised and non-randomised studies of health care interventions. J Epidemio/ Community Health 1998;52:377-84.

39 OCEBM Levels of Evidence Working Group. The Oxford levels of evidence 2. Oxford centre for evidence-based medicine, 2011. Available: https://www.cebm.ox.ac.uk/ resources/levels-of-evidence/ocebm-levels-of-evidence [Accessed 14 Jun 2020].

40 Schwarzer G. Meta: an R package for meta-analysis. $R$ news 2007;7:40-5.

41 Viechtbauer W. Conducting meta-analyses in R with the metafor package. J Stat Softw 2010;36:1-48.

42 R Core Team. R: a language and environment for statistical computing. Vienna, Austria: R Foundation for Statistical Computing, 2017.

43 Harrer M, Cuijpers P, Furukawa T. dmetar: companion R package for the guide "doing meta-analysis in R". R package version 0.0. 9000, 2019.

44 McCartney K, Rosenthal R. Effect size, practical importance, and social policy for children. Child Dev 2000;71:173-80.

45 Lipsey MW, Wilson DB. The efficacy of psychological, educational, and behavioral treatment. confirmation from meta-analysis. Am Psychol 1993;48:1181-209.

46 Borenstein M, Hedges LV, Higgins JP, et al. Introductionto meta-analysis. West Sussex, England: John Wiley\&Sons Ltd, 2009.

47 Higgins JPT TJ, Chandler J, Cumpston M. Cochrane Handbook for systematic reviews of interventions version 6.0. Cochrane, 2019. Available: www.training.cochrane.org/ handbook [Accessed 14 Jun 2020].

48 Olkin I, Dahabreh IJ, Trikalinos TA. GOSH - a graphical display of study heterogeneity. Res Synth Methods 2012;3:214-23.
49 Cuijpers P. Meta-Analyses in mental health research. A practical guide. Amsterdam, the Netherlands: Pim Cuijpers Uitgeverij, 2016.

50 Viechtbauer W, Cheung MW-L. Outlier and influence diagnostics for meta-analysis. Res Synth Methods 2010;1:112-25.

51 McKinlay A, Grace RC, Horwood LJ, et al. Long-term behavioural outcomes of preschool mild traumatic brain injury. Child Care Health Dev 2010;36:22-30.

52 Ellis MJ, Ritchie LJ, Koltek M, et al. Psychiatric outcomes after pediatric sports-related concussion. J Neurosurg Pediatr 2015;16:709-18.

53 Kontos AP, Covassin T, Elbin RJ, et al. Depression and neurocognitive performance after concussion among male and female high school and collegiate athletes. Arch Phys Med Rehabil 2012;93:1751-6.

54 Kenardy J, Le Brocque R, Hendrikz J, et al. Impact of posttraumatic stress disorder and injury severity on recovery in children with traumatic brain injury. J Clin Child Adolesc Psychol 2012;41:5-14.

55 Ho RA, Hall GB, Noseworthy MD, et al. An emotional go/no-go fMRI study in adolescents with depressive symptoms following concussion. Int J Psychophysiol 2018;132:62-73

56 Crowe L, Collie A, Hearps S, et al. Cognitive and physical symptoms of concussive injury in children: a detailed longitudinal recovery study. $\mathrm{Br} J$ Sports Med 2016;50:311-6.

57 Brooks BL, Plourde V, Beauchamp MH, et al. Predicting psychological distress after pediatric concussion. J Neurotrauma 2019;36:679-85.

58 Bloom DR, Levin HS, Ewing-Cobbs L, et al. Lifetime and novel psychiatric disorders after pediatric traumatic brain injury. J Am Acad Child Adolesc Psychiatry 2001;40:572-9

59 Anderson V, Brown S, Newitt $\mathrm{H}$, et al. Educational, vocational, psychosocial, and quality-of-life outcomes for adult survivors of childhood traumatic brain injury. $J$ Head Trauma Rehabil 2009;24:303-12.

60 Barker-Collo SL. Behavioural profiles and injury severity following childhood traumatic brain injury. Brain Impairment 2007;8:22-30.

61 Brown EA, Kenardy J, Chandler B, et al. Parent-reported health-related quality of life in children with traumatic brain injury: a prospective study. J Pediatr Psychol 2016:41:244-55

62 Rosema S, Muscara F, Anderson V, et al. The trajectory of long-term psychosocial development 16 years following childhood traumatic brain injury. J Neurotrauma 2015;32:976-83

63 Anderson VA, Catroppa C, Haritou F, et al. Identifying factors contributing to child and family outcome 30 months after traumatic brain injury in children. J Neurol Neurosurg Psychiatry 2005:76:401-8.

64 Catale C, Marique P, Closset A, et al. Attentional and executive functioning following mild traumatic brain injury in children using the test for attentional performance (TAP) battery. J Clin Exp Neuropsychol 2009;31:331-8.

65 Plourde V, Daya H, Low TA, et al. Evaluating anxiety and depression symptoms in children and adolescents with prior mild traumatic brain injury: agreement between methods and respondents. Child Neuropsychol 2019;25:44-59.

66 Truss K, Godfrey C, Takagi M, et al. Trajectories and risk factors for post-traumatic stress symptoms following pediatric concussion. J Neurotrauma 2017;34:2272-9.

67 Smyth K, Sandhu SS, Crawford S, et al. The role of serotonin receptor alleles and environmental stressors in the development of post-concussive symptoms after pediatric mild traumatic brain injury. Dev Med Child Neurol 2014;56:73-7.

68 Ho RA, Hall GB, Noseworthy MD, et al. Post-concussive depression: evaluating depressive symptoms following concussion in adolescents and its effects on executive function. Brain Inj 2020;34:520-7.

69 Butler K, Rourke BP, Fuerst DR, et al. A typology of psychosocial functioning in pediatric closed-head injury. Child Neuropsychology 1997;3:98-133.

70 Prigatano GP, Gray JA. Parental concerns and distress after paediatric traumatic brain injury: a qualitative study. Brain Inj 2007;21:721-9.

71 Ryan NP, Hughes N, Godfrey C, et al. Prevalence and predictors of externalizing behavior in young adult survivors of pediatric traumatic brain injury. J Head Trauma Rehabil 2015;30:75-85

72 Anderson VA, Catroppa C, Haritou F, et al. Predictors of acute child and family outcome following traumatic brain injury in children. Pediatr Neurosurg 2001:34:138-48

73 Bernard CO, Ponsford JL, McKinlay A, et al. Do concussive symptoms really resolve in young children? J Head Trauma Rehabil 2017;32:413-24.

74 Anderson V, Le Brocque R, Iselin G, et al. Adaptive ability, behavior and quality of life pre and posttraumatic brain injury in childhood. Disabil Rehabil 2012;34:1639-47.

75 Laliberté Durish C, Yeates KO, Brooks BL. Convergent and divergent validity of the Connor-Davidson resilience scale in children with concussion and orthopaedic injury. Brain Inj 2018;32:1525-33

76 Guo X, Edmed SL, Anderson V, et al. Neurocognitive predictors of posttraumatic stress disorder symptoms in children 6 months after traumatic brain injury: a prospective study. Neuropsychology 2017;31:84-92.

77 Bunt SC, Didehbani N, Tarkenton T, et al. Sex differences and reporting of SCAT-5 concussion symptoms in adolescent athletes. Clin J Sport Med 2020. doi:10.1097/ JSM.0000000000000788. [Epub ahead of print: 24 Jan 2020].

78 Sariaslan A, Sharp DJ, D'Onofrio BM, et al. Long-term outcomes associated with traumatic brain injury in childhood and adolescence: a nationwide Swedish 
cohort study of a wide range of medical and social outcomes. PLoS Med 2016;13:e1002103.

79 Bellerose J, Bernier A, Beaudoin C, et al. When injury clouds understanding of others: theory of mind after mild TBI in preschool children. J Int Neuropsycho/ Soc 2015;21:483-93.

80 Antshel KM, Malhotra A, Seigers D. Attributions of behavior in the pediatric mild closed head injury (chi) population. Psychol Health Med 2007;12:48-63.

81 Crowe LM, Catroppa C, Babl FE, et al. Intellectual, behavioral, and social outcomes of accidental traumatic brain injury in early childhood. Pediatrics 2012;129:e262-8.

82 Wilmoth K, Curcio N, Tarkenton T, et al. Utility of brief psychological measures for prediction of prolonged symptom clearance in concussed student athletes. Arch Clin Neuropsychol 2019. doi:10.1093/arclin/acz061. [Epub ahead of print: 26 Nov 2019].

83 Jones KM, Barker-Collo S, Parmar P, et al. Trajectories in health recovery in the 12 months following a mild traumatic brain injury in children: findings from the bionic study. J Prim Health Care 2018;10:81-9.

84 Kaldoja M-L, Kolk A. Social-emotional behaviour in infants and toddlers with mild traumatic brain injury. Brain Inj 2012;26:1005-13.

85 Ryan NP, Noone K, Godfrey C, et al. Young adults' perspectives on health-related quality of life after paediatric traumatic brain injury: a prospective cohort study. Ann Phys Rehabil Med 2019;62:342-50.

86 Zemek R, Clarkin C, Farion KJ, et al. Parental anxiety at initial acute presentation is not associated with prolonged symptoms following pediatric concussion. Acad Emerg Med 2013;20:1041-9.

87 DiFazio M, Silverberg ND, Kirkwood MW, et al. Prolonged activity restriction after concussion: are we worsening outcomes? Clin Pediatr 2016;55:443-51.

88 Silverberg ND, Iverson GL. Is rest after concussion "the best medicine?": recommendations for activity resumption following concussion in athletes, civilians, and military service members. J Head Trauma Rehabil 2013;28:250-9.

89 Sady MD, Vaughan CG, Gioia GA. Psychometric characteristics of the postconcussion symptom inventory in children and adolescents. Arch Clin Neuropsychol 2014;29:348-63.

90 Yaramothu C, Goodman AM, Alvarez TL. Epidemiology and incidence of pediatric concussions in general aspects of life. Brain Sci 2019;9:257.

91 Valovich McLeod TC, Wagner AJ, Bacon CEW. Lived experiences of adolescent athletes following sport-related concussion. Orthop J Sports Med 2017; 5:232596711774503.

92 Rice SM, Parker AG, Rosenbaum S, et al. Sport-related concussion and mental health outcomes in elite athletes: a systematic review. Sports Med 2018;48:447-65.

93 Fralick M, Sy E, Hassan A, et al. Association of concussion with the risk of suicide: a systematic review and meta-analysis. JAMA Neurol 2019;76:144-51.
94 Königs M, van Heurn LWE, Vermeulen RJ, et al. Feedback learning and behavior problems after pediatric traumatic brain injury. Psychol Med 2016;46:1473-84.

95 Königs M, Heij HA, van der Sluijs JA, et al. Pediatric traumatic brain injury and attention deficit. Pediatrics 2015:136:534-41.

96 Renström B, Söderman K, Domellöf E, et al. Self-reported health and influence on life situation 5-8 years after paediatric traumatic brain injury. Brain Inj 2012;26:1405-14.

97 Stojanovski S, Felsky D, Viviano JD, et al. Polygenic risk and neural substrates of attentiondeficit/hyperactivity disorder symptoms in youths with a history of mild traumatic brain injury. Biol Psychiatry 2019;85:408-16.

98 Anderson V, Godfrey C, Rosenfeld JV, et al. 10 years outcome from childhood traumatic brain injury. Int J Dev Neurosci 2012;30:217-24.

99 Giza CC, Kutcher JS, Ashwal S, et al. Summary of evidence-based guideline update: evaluation and management of concussion in sports: report of the Guideline Development Subcommittee of the American Academy of Neurology. Neurology 2013;80:2250-7.

100 Reed N, Zemek R, Dawson J. Living guideline for diagnosing and managing pediatric concussion, 2019.

101 Leddy JJ, Willer B. Use of graded exercise testing in concussion and return-to-activity management. Curr Sports Med Rep 2013:12:370-6.

102 Schneider KJ, Meeuwisse WH, Nettel-Aguirre A, et al. Cervico-vestibular physiotherapy in the treatment of individuals with persistent symptoms following sport related concussion: a randomised controlled trial. Br J Sports Med 2013;47:e1.50-e1.

103 Gagnon I, Grilli L, Friedman D, et al. A pilot study of active rehabilitation for adolescents who are slow to recover from sport-related concussion. Scand I Med SCi Sports 2016;26:299-306.

104 McCarty CA, Zatzick DF, Marcynyszyn LA, et al. Effect of collaborative care on persistent Postconcussive symptoms in adolescents: a randomized clinical trial. JAMA Netw Open 2021;4:e210207-e.

105 Stein E, Howard W, Rowhani-Rahbar A, et al. Longitudinal trajectories of postconcussive and depressive symptoms in adolescents with prolonged recovery from concussion. Brain Inj 2017;31:1736-44.

106 Lawrence D, Johnson S, Hafekost J, et al. The mental health of children and adolescents: report on the second Australian child and adolescent survey of mental health and wellbeing. Canberra: Department of Health, 2015.

107 Rockhill CM, Fann JR, Fan M-Y, et al. Healthcare costs associated with mild traumatic brain injury and psychological distress in children and adolescents. Brain Inj 2010;24:1051-60.

108 Durish CL, Yeates KO, Brooks BL. Psychological resilience as a predictor of symptom severity in adolescents with poor recovery following concussion. J Int Neuropsychol Soc 2019:25:346-54. 